\title{
BLACK-HOLE ACCRETION CORONA IMMERSED IN THE DISK RADIATION FIELDS
}

\author{
T. MIWA, Y. WATANABE, J. FUKUE \\ Astronomical Institute, Osaka Kyoiku University, \\ Asahigaoka, Kashiwara, Osaka 582, Japan
}

\section{Accretion Corona}

We examined an accretion-disk corona around a black hole immersed in the disk radiation fields (cf. Watanabe, Fukue 1996a, b). The corona is supposed to be initially at rest far from the center. During infall above and below the disk, the corona is suffered from the disk radiation fields. As a disk model, we adopted the standard $\alpha$-disk, and in order to mimic the general relativisitic effects, we use the pseudo-Newtonian force proposed by Artemova et al. (1996). Moreover, we assume that the corona is geometrically thin and optically thin, and ignored any motion such as wind. We consider the cold case, where the pressure-gradient force is ignored. Under these assumptions, we calculated the motion of the corona gas and found that the infall of corona is supressed due to disk radiation fields.

\section{Results}

The results are shown in the following figures. If the disk luminosity increases, the radial infall velocity of the corona gas deviates from the free-fall one and becomes small, since the corona gas gains the angular momentum from the underlying disk via radiation drag of disk radiation fields. When the disk is sufficiently luminous, the infalling of the gas is stopped (in such a case, the corona gas may be blown off from the disk).

These properties are summarized in the $\Gamma_{\mathrm{d}^{-}} a$ plane, where $a$ is the spin parameter of the hole and $\Gamma_{d}$ is the normalized disk luminosity (= $\left.L_{\mathrm{d}} / L_{\mathrm{Edd}}\right)$. The corona gas can infall when $\Gamma_{\mathrm{d}}$ is smaller than some critical values, and this critical $\Gamma_{\mathrm{d}}$ becomes small, as $a$ increases. 

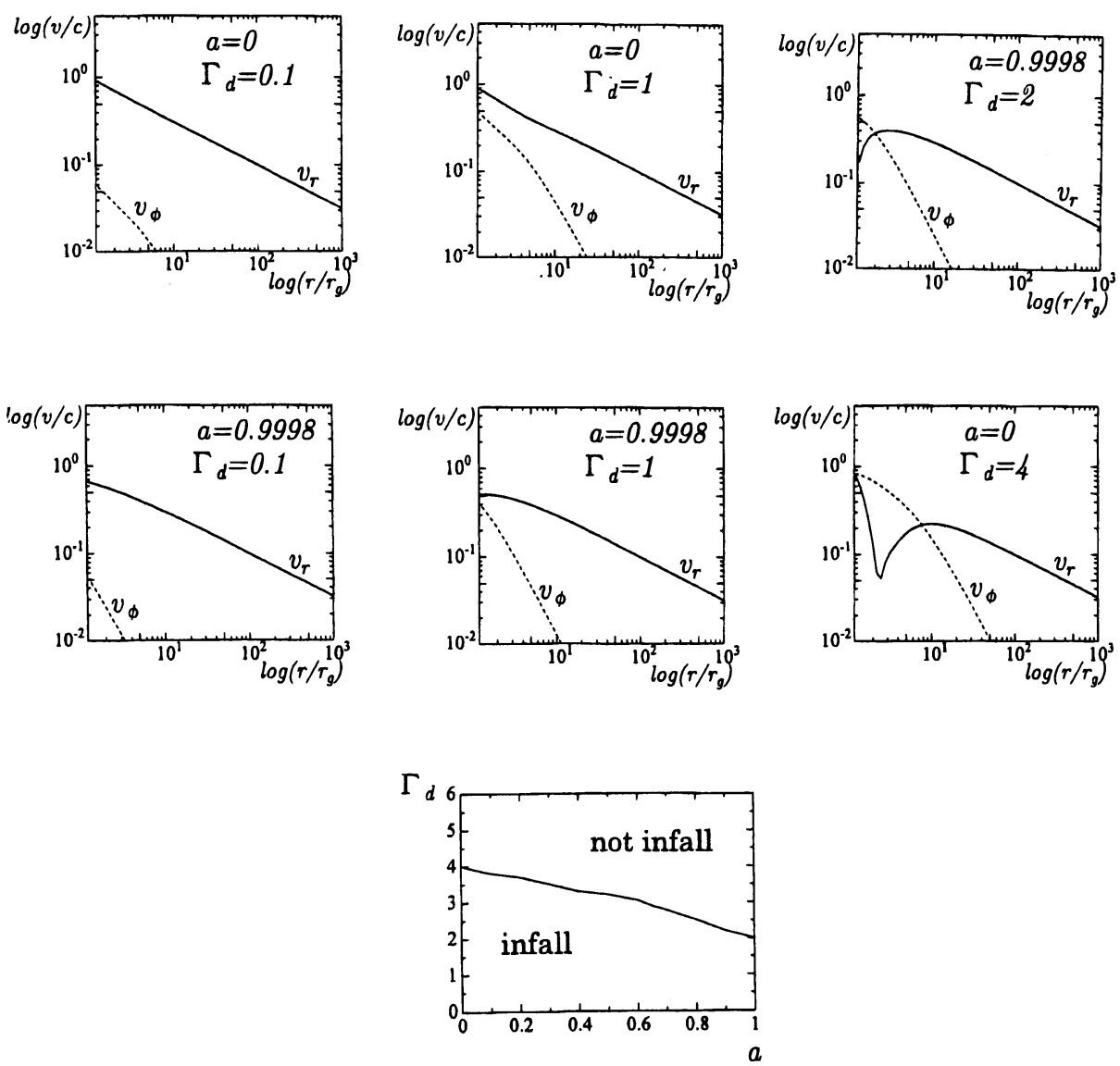

\section{Conclusion}

When we consider the normal (proton-electron) plasmas, the corona gas can always infall, since $\Gamma_{d}$ does not exceed unity. The infall velocity, however, deviates from the free-fall one due to the radiation drag of disk radiation fields. When we consider electron-positron plasmas, however, the infall will be remarkably modified, since $\Gamma_{d}$ can exceed unity $\left(\Gamma_{d} \sim 1836\right)$. In this case where $\Gamma_{d}$ becomes so large, the corona gas will escape as radiatively driven winds (Tajima, Fukue 1996).

\section{Reference}

Artemova I. V., Björnsson G., Novikov I. D. 1996, ApJ 461, 565

Tajima Y., Fukue J. 1996, PASJ 48, 529

Watanabe Y., Fukue J. 1996a, PASJ 48, 841

Watanabe Y., Fukue J. 1996b, PASJ 48, 849 